\title{
Does dietary knowledge in patients on haemodialysis influence compliance?
}

\author{
D. Khawnekar ${ }^{1}$, Y. M. Jeanes ${ }^{1}$, E. L. Gibson ${ }^{1}$, I. Held ${ }^{2}$ and P. Rutherford ${ }^{2}$ \\ ${ }^{1}$ School of Human \& Life Sciences, Roehampton University, London SW15 4JD, UK and ${ }^{2}$ Renal Unit, \\ Wrexham Maelor Hospital, Wrexham LL13 7TD, UK
}

Dietary restriction of $\mathrm{K}$, phosphate and fluid are commonplace in patients with end-stage renal failure. Previous studies have shown that patients on haemodialysis (HD) exhibit poor dietary compliance ${ }^{(1)}$. The aim of the present study is to explore knowledge of food sources and dietary compliance in patients on HD.

Thirty-nine patients attending a HD unit completed the study over four consecutive weeks in 2007; they were on HD for $>3$ months and had received advice from the renal dietitian. Patients completed a modified twenty-six-item renal knowledge questionnaire ${ }^{(2)}$. Adherence to the dietary restrictions was monitored by serum K, phosphate and inter-dialytic weight gain (IDWG) over the previous three 1-month periods.

The patients were aged between 25 and 85 years (twenty-six males and thirteen females). The length of time on HD ranged from 3 months to 16 years. Twenty-three (59\%) patients reported fully understanding the dietary advice and sixteen (41\%) requested further dietary advice, predominately relating to $\mathrm{K}$ and phosphate.

\begin{tabular}{|c|c|c|c|c|c|c|c|}
\hline \multicolumn{8}{|c|}{ Correct responses to questions relating to foods high in: } \\
\hline \multicolumn{2}{|c|}{$\mathrm{K}$} & \multicolumn{2}{|c|}{ Phosphate } & \multicolumn{2}{|c|}{$\mathrm{Na}$} & \multicolumn{2}{|c|}{ Fluid } \\
\hline$n$ & $\%$ & $n$ & $\%$ & $n$ & $\%$ & $n$ & $\%$ \\
\hline 26 & 67 & 21 & 54 & 29 & 74 & 29 & 74 \\
\hline
\end{tabular}

Patients were most knowledgeable about foods high in Na and fluid. Patients varied in their compliance: K, $n$ 24; phosphate, $n$ 26; fluids, $n$ 18. Knowledge of food sources high in $\mathrm{K}$ or phosphate did not impact on whether they were compliant $(P>0.05)$. Knowledge of foods high in $\mathrm{Na}$ or fluid also did not influence compliance, although there were more patients who were non-compliant based on IDWG who had poor knowledge of Na-containing foods (ten $v$. five).

Patients had a variable knowledge of foods containing high levels of electrolytes and fluids, which highlights a potential need for reinforced dietary advice. However, there was no clear relationship between knowledge of food sources high in electrolytes and fluids and dietary compliance, in agreement with previous findings ${ }^{(2)}$. The present findings suggest other factors, such as stress, health locus of control, and personality, may have a greater impact on dietary compliance when compared with knowledge of food sources. However, it should be noted that a certain level of knowledge must be required to enable the patients to choose appropriate foods within the renal diet. However, common psychological factors may mediate both knowledge and compliance and further investigation into patients with poor dietary knowledge is warranted. Future studies are needed to help elucidate what factors influence dietary compliance, and thus health outcomes, in patients on HD.

1. Kugler C, Vlaminck H, Haverich A et al. (2005) J Nurs Scholarsh 37, 25-29.

2. Durose CL, Holdsworth M, Watson V et al. (2004) J Am Diet Assoc 104, 35-41. 